\title{
Visual Feedback on Nonverbal Communication: A Design Exploration with Healthcare Professionals
}

\author{
Rupa A. Patel, Andrea Hartzler, \\ Wanda Pratt, Anthony Back \\ University of Washington \\ Seattle, Washington, USA \\ \{rupatel, andreah, wpratt, tonyback\}@uw.edu
}

\author{
Mary Czerwinski, Asta Roseway \\ Microsoft Research \\ Redmond, Washington, USA \\ \{marycz, astar\}@microsoft.com
}

\begin{abstract}
Effective nonverbal communication between patients and clinicians fosters both the delivery of empathic patientcentered care and positive patient outcomes. However, few efforts to develop tools for enhancing clinician communication have focused on nonverbal aspects of the clinical encounter. We describe a novel system that both uses social signal processing technology (SSP) to capture nonverbal cues in real time and displays ambient visual feedback on control and affiliation-two primary, yet distinct dimensions of interpersonal nonverbal communication. To explore clinicians' acceptance of and reaction to an ambient visual feedback from such a system, we conducted a Wizard-of-Oz lab study to simulate system use with 16 healthcare professionals. We further followed up with 7 of those participants and iterated on the design with a new visualization. Our results indicate that reflective visual feedback on nonverbal communication provides an acceptable way to provide clinicians with awareness of their nonverbal communication. Furthermore, we discuss implications for the design of visual feedback to encourage empathic patient-centered communication.
\end{abstract}

Keywords-nonverbal communication; patient-clinician communication; user-centered design; iterative design

\section{INTRODUCTION}

In addition to speaking clearly and avoiding jargon, skilled "bedside manner" requires nonverbal competencies. The ability to understand and convey nonverbal signals is essential to forming empathic relationships in patient-centered care [1]. Specifically, nonverbal cues, such as voice tone, body movement, and facial expression, link to important patient outcomes [2], such as patient satisfaction [3] and adherence to medication [4]. Traditional clinical communication training lacks specificity on these nonverbal competencies, using directives such as "offer empathy in greeting" [5]. Because such training typically takes place outside the context of the clinical encounter, clinicians must struggle to transfer skills from training or to learn new skills at the point of care. Given time constraints on clinical care delivery and the difficulty of teaching such skills outside the context of care, improving the nonverbal communication skills of clinicians remains a grand challenge [5].

We take steps to address this challenge through the development of a system called Entendre [6]. Entendre is designed to display ambient visual feedback on nonverbal cues. In this work, we focus on use by health professionals and their clinical encounters with patients. The ultimate goal of this feedback is to enhance clinicians' self-awareness of their nonverbal communication. Entendre is designed to produce visual feedback from real-time social signal processing of a video feed from two people in conversation [6].

For this work, we explored the visual design of feedback Entendre presents. We hope to engage clinicians in improving their nonverbal communication without distracting from patient care. We address the following research questions (RQ's):

$R Q 1$. Is real-time ambient visual feedback on nonverbal communication acceptable to clinicians?

RQ2. What are design considerations of such ambient visual feedback developed for clinicians?

We answered these research questions through two formative design studies. First, we conducted a Wizard-of-Oz lab study in which we obtained feedback on an ambient realtime visualization from 16 healthcare professionals. We subsequently followed up with a new design and interviews with 7 of the same participants. Our findings generate implications to consider when designing visual feedback for clinicians regarding their nonverbal communication skills.

\section{RELATED WORK}

Social signal processing technologies (SSP) perform "automatic sensing and interpretation of social signals, which are complex aggregates of nonverbal behaviors through which individuals express their attitudes towards other human (and virtual) participants in the current social context" [7]. Thus, SSP detects nonverbal behavioral cues as communicative signals in social interactions. Researchers have developed SSP systems that use machine learning to identify nonverbal cues. In Pentland's work, sociometer badges consist of sensors that automatically classify specific groups of nonverbal cues (e.g., physical proximity, pitch variation) into one of four "honest signals": activity, consistency, influence, and mimicry [8]. For example, an individual matching the pitch and speech rate of a conversational partner is an instance of "mimicry." In later work, Byun et al. augmented a videoconferencing system to identify nonverbal cues without using physical sensors, also classifying these cues into honest signals [9]. We extend this work by grounding our nonverbal feedback in studies of relational communication $[10,11,12]$, particularly between clinicians and patients $[13,14]$. 
TABLE I. RELATIONSHIP OF NONVERBAL CUES TO CONTROL AND AFFILIATION

\begin{tabular}{|l|c|c|c|c|c|c|}
\hline \multirow{2}{*}{ NONVERBAL CUE } & \multicolumn{3}{c|}{ AFFILIATION } & \multicolumn{3}{c|}{ CONTROL } \\
\cline { 2 - 7 } & Rapport & Trust & Warmth & Dominance & Influence & Authority \\
\hline Nodding (positive reinforcer) & + & + & + & $(+)$ & & $(+)$ \\
\hline Head shaking (agreement) & & & & & & $(+)$ \\
\hline Varied pitch & + & $(+)$ & & & $(+)$ & \\
\hline Varied tempo & + & $(+)$ & & & $(+)$ & \\
\hline Increased talk time (talk time) & & & & $(+)$ & & $(+)$ \\
\hline Interruption (barge-in) & & & & & & \\
\hline Interruption (barge-in+suppress) & & & & + & & \\
\hline
\end{tabular}

Legend

\begin{tabular}{|c|l|}
\hline+ & $\begin{array}{l}\text { Evidence of positive } \\
\text { relationship clinically }\end{array}$ \\
\hline$(+)$ & $\begin{array}{l}\text { Evidence of positive } \\
\text { relationship generally }\end{array}$ \\
\hline & Favorable outcome \\
\hline & Unfavorable outcome \\
\hline & Mixed outcome \\
\hline
\end{tabular}

Researchers have also used SSP to influence interactions in small groups through both public and private displays of behavior-based feedback. Using the Meeting Mediator system, researchers utilized sociometer badges and mobile displays to influence overlapping speaking time and increase interactivity level in small group meetings [15]. Other researchers used a peripheral display to promote awareness of speaking time and eye gaze in small groups to encourage collaboration [16]. Vocal features, body language cues, and physiological signals have fed into such automated feedback displays. Evaluations indicated that realtime feedback on speaking activity could balance participation among meeting members. In both projects, design implications from visual elements of the feedback were not explored in depth.

Although use of SSP has not been explored yet in the clinical setting, one research tool, Discursis, employs natural language processing for clinician communication training [17]. Discursis takes as input transcripts of patient encounters and produces a visual representation of the conceptual content and turn-taking dynamics of a clinical encounter. The researchers studied both the fulfillment of clinical duties and the rapportbuilding that the clinicians engaged in, based on a text analysis of the transcripts [17]. Discursis assesses clinicians' verbal communication skills after the fact for awareness, whereas our approach addresses clinicians' nonverbal skills in real time.

In the patient-clinician communication literature, researchers have used self-report and observational methods to assess empathy and nonverbal communication. One approach relies on the patient to rate how empathic the clinician was during an encounter [18]. Alternatively, observational methods involve third-party observers watching videos of clinical encounters and either labeling the nonverbal cues [19] or assigning global ratings, such as overall affect [20]. Labeling cues requires considerable time and training. Thus, researchers might label only a "thin slice" of the clinic encounter and attempt to make inferences about clinicians' overall communication skill [19]. With SSP, we can move beyond the limitations of self-report questionnaires, ratings that require trained observers during a clinic encounter, and labor-intensive coding processes.

\section{RELATIONAL COMMUNICATION FRAMEWORK}

We devised a framework that maps nonverbal cues to concepts in empathic patient-centered communication [6]. We drew upon a validated model of relational communication that has been applied both in clinical settings $[13,14]$ and more broadly to general interpersonal communication $[10,11$. 12]. Two primary types of relational signaling-affiliation (reflecting interpersonal warmth, trust, and rapport) and control (reflecting dominance, influence, and authority) — make up core dimensions of nonverbal communication. Affiliative and more controlling styles are prevalent communication patterns exhibited by clinicians $[13,14]$. Table 1 lists relationships of select nonverbal captured by Entendre to concepts related to control and affiliation. More detail can be found in [6]0. We anticipate that the ambient visual feedback produced from categorizing combinations of these nonverbal cues can encourage empathic patient-centered communication.

\section{FORMATIVE LAB STUDY}

We explored the acceptability and design considerations of ambient, real-time, nonverbal communication feedback for clinicians. We conducted a Wizard-of-Oz (WOZ) study to focus our data collection on both real-time and conceptual properties of the visual feedback, in addition to exploring the impact of design decisions that we made.

\section{A. Initial Feedback Design}

Our initial visualization juxtaposed two separate visual elements representing affiliation and control, which each changed in real-time (Figure 1). A sun-moon, represented a 7point measure of affiliation. This element was a small, cool blue moon (Figure 1b) when affiliation was lowest, increasing in size and becoming warmer in color as affiliation increased. At the highest level of affiliation, the element was a large, yellow sun increases (Figure 1a). In our study, a researcher controlled the feedback level in response to observing the nonverbal cues. For example, when the health professional expressed signs of affiliation, such as leaning toward the patient and nodding, the researcher increased the affiliation level and the visual element turned sun-like by becoming larger and more yellow in color. Concurrently, the lower visual element included a seesaw, representing a 7-point measure of control. Conversational control was represented by the ball rolling to the left side near the health professional (i.e., "You") when the health professional dominated and rolling to the right side near the patient's name (i.e., "Alicia") when the patient dominated the conversation.

\section{B. Methods}

We conducted a role-play scenario with 16 healthcare professionals to gather their perceptions and acceptance of ambient, real-time visual feedback. The task for each participant was to be as empathic as possible with an actor playing the role of a patient and to incorporate feedback from the ambient visual display as much as $\mathrm{s} /$ he chose. Participants engaged with a professional actor who had improvisation experience simulating a patient in medical school exams. We 


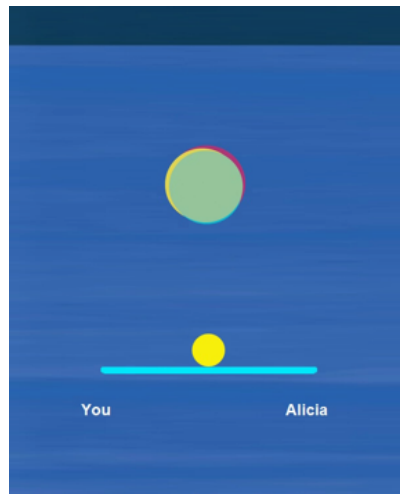

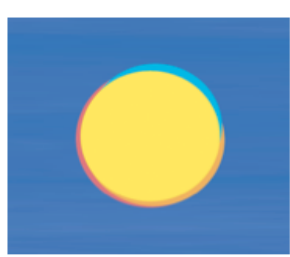

(a) Affiliation $=7$

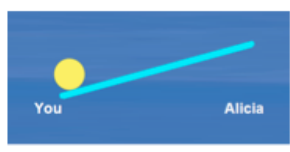

(c) Control $=7$

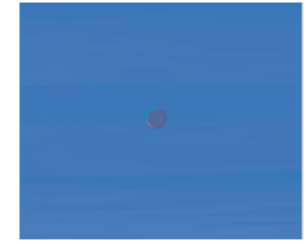

(b) Affiliation $=1$

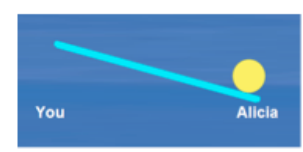

(d) Control $=1$
Figure 1. Sun-moon/seesaw design. Large, yellow sun (a) indicates high affiliation. Small, blue moon (b) indicates low affiliation. Seesaw tipped to participant's name "You" (c) indicates high control. Seesaw tipped (d) to actor's name "Alicia" indicates low control.

designed a non-clinical counseling scenario to enable recruitment from a broad range of health professions. Potential participants were screened for prior volunteer counseling experience to help ensure natural role-play setup. We set up two pilot sessions with people from the general population to train the actor and modify the scenario to help make the task more natural.

(1) Participants: We recruited 16 healthcare professionals whose specialties are listed in Table 2. Participants ranged in age from 25-55 (mean 40.3, median 42), and nine were men. We selected young to middle-aged healthcare professionals to obtain a perspective from those who are used to technology in their everyday lives. All participants were compensated via software gratuity.

TABle II. PARTICIPANTS' PROFESSIONAL SPECIALTIES

\begin{tabular}{|l|c|}
\hline \multicolumn{1}{|c|}{ Professional Specialty } & Number of Participants \\
\hline Emergency Medical Technician (EMT) & 5 \\
\hline Nurse & 4 \\
\hline Certified Nursing Assistant (CNA) & 2 \\
\hline Speech-Language Pathologist & 2 \\
\hline Physician & 1 \\
\hline Dentist & 1 \\
\hline Chiropractor & 1 \\
\hline
\end{tabular}

(2) Study Procedures: Prior to leading participants to the observation room, the researcher played a training video and gave them a handout that explained the meaning of the visual feedback. The handout described the concepts of control and affiliation, and what cues mapped to high and low demonstrations of these concepts. The researcher also shared role-play instructions for an initial "getting-to-know-you" counseling meeting with a shy young woman, played by the actor. Participants were free to incorporate details from their own lives. The actor was the same for all participants to keep the scenario experience consistent. We did not inform the actor of specific goals or expected outcomes. Finally, the researcher did not reveal that she-rather than the SSP-would be generating the visual feedback.

The observation room was set up as shown in Figure 2. Two auxiliary video cameras captured the head and shoulders

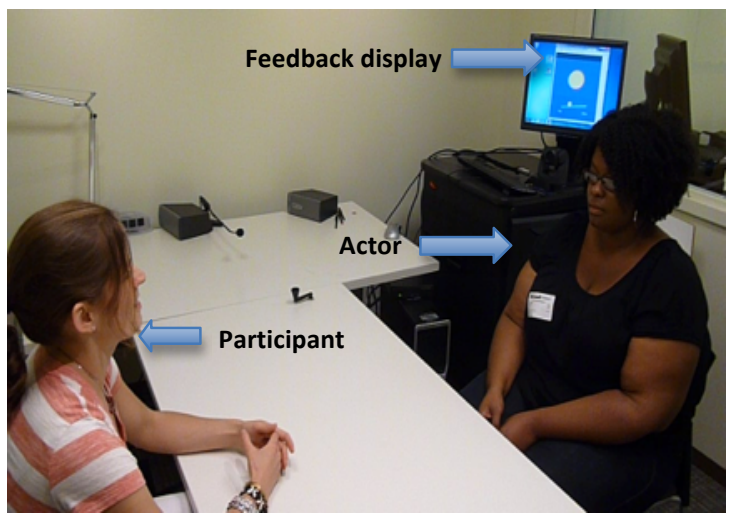

Figure 2. Observation room setup.

of the participant and of the actor. The first 8 participants received visual feedback from a 17" display placed on the table two feet to the left in between both parties, tilted towards the participant. The last 8 participants received visual feedback from the display placed two feet behind the actor and slightly to the left (as shown in Figure 2). In both cases, the actor could not view the display from where she was sitting.

The role-play conversation lasted 10-12 minutes, with the display showing no feedback for the initial $2 \frac{1}{2}$ minutes. We created this initial no-feedback period to help the participants get immediately acquainted with the actor without distractions. The researcher controlling the visual feedback referred to a live video stream of the participant, the actor, and the feedback display. She rated the affiliation and control of participants on a 7-point scale, basing her overall judgment on cues such as interruption, speech rate, nodding, and pitch variation (Table 1). On average, the researcher updated the feedback every 6.5 seconds. We logged the timestamps of changes the researcher made to the control and affiliation levels in a database for later analysis.

After the role-play conversation was over, the researcher administered questionnaires to both the health professional participant and actor. In a brief exit interview, the researcher also asked for further comments on participants' attitude towards the technology and its impact on the interaction.

(3) Study Data: Our data collection included assessments of consistency of the feedback, acceptability by clinicians, and its perceived impact on the participants' manner.

(a) Feedback Consistency: The actor completed the widely-used Consultation and Relational Empathy Measure (CARE) [18] that we slightly modified to describe a nonclinical meeting. We used CARE to assess the actor's perception of the participant's empathy and to compare these scores with researcher-controlled feedback. We assessed the relationship between CARE empathy scores and average control and affiliation levels (weighted by time at each level) using a Pearson's correlation.

(b) Acceptability: The questionnaire administered to particpants captured the following: 
- Likes and dislikes for both sun-moon representation of affiliation and seesaw representation of control (open-ended)

- Design feedback on a 5-point Likert scale about how informative, interesting, distracting, helpful, and confusing the participant found the visualization

- Whether participants would use the technology in a professional setting (yes/no/unsure)

The exit interview included open-ended feedback on:.

(c) Perceived Impact on Participants' Manner: We asked participants how they thought the feedback display influenced their behavior with the actor. In particular:

- Self-reported frequency of glancing at the visualization (1-2 times, 3-6 times, every minute, more than once per minute)

- Perceived effect of the visualization on participants' manner (open-ended)

\section{Results}

\section{(1) Feedback Consistency}

There is a positive correlation between the participants' CARE scores and their average affiliation levels (Pearson's $r=$ $0.815, \mathrm{p}<.001)$. However, no relationship exists between CARE scores and control levels. Still, the actor engaged participants enough to make the role-play feel realistic for the participants, which was consistently expressed in exit interviews.

\section{(2) Acceptability}

(a) Likes and dislikes: The majority of participants had a positive attitude towards the visual feedback, although participants disliked a few aspects of the feedback. We grouped participants' similar likes and dislikes for both control and affiliation graphical elements in Table 3. Despite the relatively short time with the visual feedback, 4 participants said that they found the visual feedback easy to interpret. The concept of control mapping to a seesaw resonated with participants who experienced the visual feedback display that was placed closer to them. Half of these participants thought that the feedback on control provided awareness of how the conversation was going. Furthermore, 4 participants commented that they thought a specfic element of the feedback was actionable.

Participants also specified a wide range of aspects that they disliked. For 3 of the 8 participants who glanced at the closer feedback display, the feedback was seen as distracting and took their attention away from the actor. This could mean that closer display placement could be more distracting, though one participant said the display was too far to the left to be usable for him while still engaging in the conversation. The timing of the update also served as a distraction to some, in particular with the real-time update of the seesaw representing control. P15 said that he felt like he had trouble finishing his speaking turn when the seesaw tilted towards him. Four participants said they had difficulty interpreting the feedback and acting on it, unsure what to do to make the graphical elements change color or size. Two participants said that they were not able to act as naturally in the presence of the feedback. All in all, we decided to iterate on the feedback to see if we could make the visual design more liked and usable.

TABLE III. LIKED AND DISLIKED ASPECTS OF INITIAL FEEDBACK

\begin{tabular}{|c|c|}
\hline & LIKES \\
\hline & $\begin{array}{l}\text { Feedback was easy to interpret } \\
\text { "Easy to quickly understand; sun and moon are good representations" } \\
\text { (P13), "Simplicity of feedback" (P7), "Easy to understand concept" (P14) }\end{array}$ \\
\hline & $\begin{array}{l}\text { Provided awareness during conversation } \\
\text { "I could tell if one of us was talking too much" (P8), "Made me aware if } \\
\text { not listening enough" (P11), "I liked the reminder to not dominate the } \\
\text { entire conversation" (P9), "Liked knowing I was being warm" }\end{array}$ \\
\hline & $\begin{array}{l}\text { Feedback was actionable } \\
\text { "Helped me to not talk too much" (P8), "Helped make sure I was was } \\
\text { engaging", "Gives visual feedback that can be quickly acted upon" (P4), } \\
\text { "Let me know that the conversation was going okay" (P1), "Told me } \\
\text { when to ask more personal questions to draw her out" (P11) }\end{array}$ \\
\hline & $\begin{array}{l}\text { Change was easy to detect } \\
\text { "Could see size change out of corner of eye" (P9), "Shows clearly the } \\
\text { balance" (P10) }\end{array}$ \\
\hline & $\begin{array}{l}\text { Didn't pay attention to it } \\
\text { "Didn't really notice it" (P5), "Didn't watch much" (P12) }\end{array}$ \\
\hline & $\begin{array}{l}\text { No likes } \\
\text { "Not much" (P16), "N/A" (P8) }\end{array}$ \\
\hline & DISLIKES \\
\hline & $\begin{array}{l}\text { Drew attention away from other person } \\
\text { "If I looked too much, it felt like I wasn't paying attention to her" (P16), } \\
\text { "Made me work at keeping eye contact" (P11), "[Kind of] distracting" } \\
\text { (P3, P5, P6), }\end{array}$ \\
\hline & $\begin{array}{l}\text { Hard to interpret } \\
\text { "Wasn't sure how to get it to [change]" (P8, P9), "Didn't like it, a little } \\
\text { vague" (P10), "Didn't understand all the colors" (P3) }\end{array}$ \\
\hline & $\begin{array}{l}\text { Caused nervousness } \\
\text { "Made me a little nervous" (P12), "Really hard to know what to do to } \\
\text { control the conversation and still be natural" (P14) }\end{array}$ \\
\hline & $\begin{array}{l}\text { Placement of monitor to one side } \\
\text { "Monitor too far to the left" (P7) }\end{array}$ \\
\hline & $\begin{array}{l}\text { Hard to see } \\
\text { "Could be bigger" (P10),"Feedback was small" (P12) }\end{array}$ \\
\hline & $\begin{array}{l}\text { Delayed timing of feedback update } \\
\text { "There was a bit of a time delay", "The quickness of it" (P13) }\end{array}$ \\
\hline & $\begin{array}{l}\text { Feedback lacked credibility } \\
\text { "At times, it didn't seem reflect what was going on" (P2) }\end{array}$ \\
\hline & $\begin{array}{l}\text { Discouraged finishing speaking turn } \\
\text { “...as soon as I started talking the seesaw would tilt towards my being } \\
\text { dominant” (P13), "When I noticed it was on me, I felt I needed to stop } \\
\text { talking and not necessarily finish topic" (P15) }\end{array}$ \\
\hline & $\begin{array}{l}\text { No dislikes } \\
\text { "Can't say there was anything I didn't like" (P15) }\end{array}$ \\
\hline
\end{tabular}

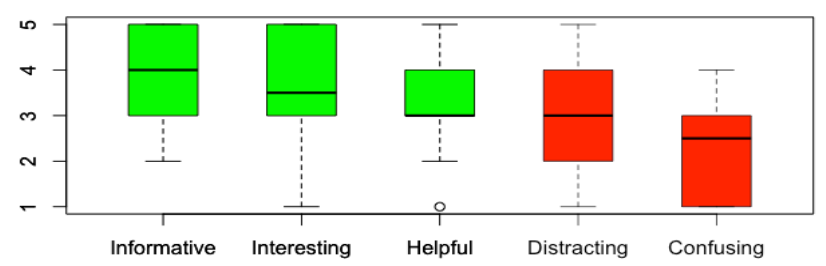

Figure 3. Subjective measures of design feedback (mean and standard error of 1-5 Likert scale scores). Green shaded boxplots are positive measures, red are negative 
(b) Design Feedback: We surveyed participants on the extent to which they found the overall visual feedback informative, interesting, helpful, distracting, and confusing, on a 5-point Likert scale, with $1=$ low and $5=$ high. Figure 3 shows that mean scores for the positive measures (Informative: 3.8, Interesting: 3.6, Helpful: 3.3) were higher than the negative measures (Distracting: 3.0, Confusing: 2.3). However, participants were polarized in their reactions.

(c) Whether participants would use the technology in a professional setting: We asked participants if they would use the tool in a professional healthcare setting. Ten participants $(63 \%)$ said yes, while only 2 participants $(13 \%)$ said no, and 4 participants $(25 \%)$ were unsure. In the brief exit interview, several participants further validated their desire to use such a tool. A physician noted that this was "something I could get used to since I'm already referring to monitors and clocks [in a clinical setting]" (P14). A nurse confirmed that this tool could be helpful because "when patients tell their story, time pressure often makes you less empathic in real situations" (P11). Still, one certified nursing assistant was more ambivalent about the visualization, saying that it "feels so subjective" (P16). Ultimately, participants confirmed our idea that there was potential for targeting clincians' improvement of nonverbal communication through visual feedback.

\section{(3) Perceived impact on participants' manner}

(a) Frequency of glancing at the visualization: Seven participants $(44 \%)$ reported that they glanced at the visualization 3-6 times and 5 participants (31\%) reported they did so every minute or more. At the low end, 4 participants $(25 \%)$ glanced at the visualization only 1-2 times.

(b) Self-reported impact on participants' manner: In their open-ended responses, 8 participants $(50 \%)$ indicated that the feedback had a positive effect on interaction with the actor, while $3(19 \%)$ thought that the effect was negative, and 5 $(31 \%)$ reported little or no perceived effect on the interaction.

\section{Design Implications}

Based on lab study findings, we generated a number of design considerations for the next iteration, including: (1) update rate, (2) position of display, (3) color, and (4) visual metaphor.

(1) Update rate. Participants were divided about how distracting they found the visualization, and this could be due to the frequent update rate (every 6.5 second on average). In the next iteration, we thought it would be best to have a slower update rate. In this way, we would allow those who found it distracting to adapt to a subtler refresh.

(2) Position of the display. Compared to first 8 participants with the display close to them, the last 8 participants who had the display behind the actor were more likely to say that the visualization was a little small and harder to see. We thought a new visualization with that explored changes to size and position of the feedback display could help illuminate what the ideal feedback size and form factor should be.
(3) Color. At least three participants said that it was difficult to detect and interpret the meaning of the colors for the sun-moon, which changed simultaneously with size. In addition, P16 said that the sun-moon color change was easy to see when it was big. We wanted to make sure that the color changes were easy to detect. As a result, we decided to vary the saturation of a single color along a gradient in the new iteration of the design.

(4) Visual metaphor. Our original design displayed control and affiliation values as separate graphical elements. Many participants found the seesaw tilt a particularly straightforward way to understand who was dominating the conversation. Still, we realized that the judgment of good vs. bad might not be appropriate in clinical settings, because during parts of clinical encounters, the clinician needs to provide treatment information rather than draw out patient history. Furthermore, P7, a dentist, commented that keeping track of two graphical elements was challenging for him since it was difficult to focus on and gauge the meaning of two separate elements with each glance. In the next iteration, we decided to combine control and affiliation measures into a composite element to reduce cognitive load.

\section{FOLlOWUP INTERVIEW STUDY}

The purpose of this follow up study was to iterate on design implications that emerged from the lab study.

\section{A. New Feedback Design}

For comparison with the initial seesaw and sun-moon visualization, we selected a lotus flower to represent control and affiliation in an artistic composite graphical element. We also wanted to test the idea of visualizing the current state of a
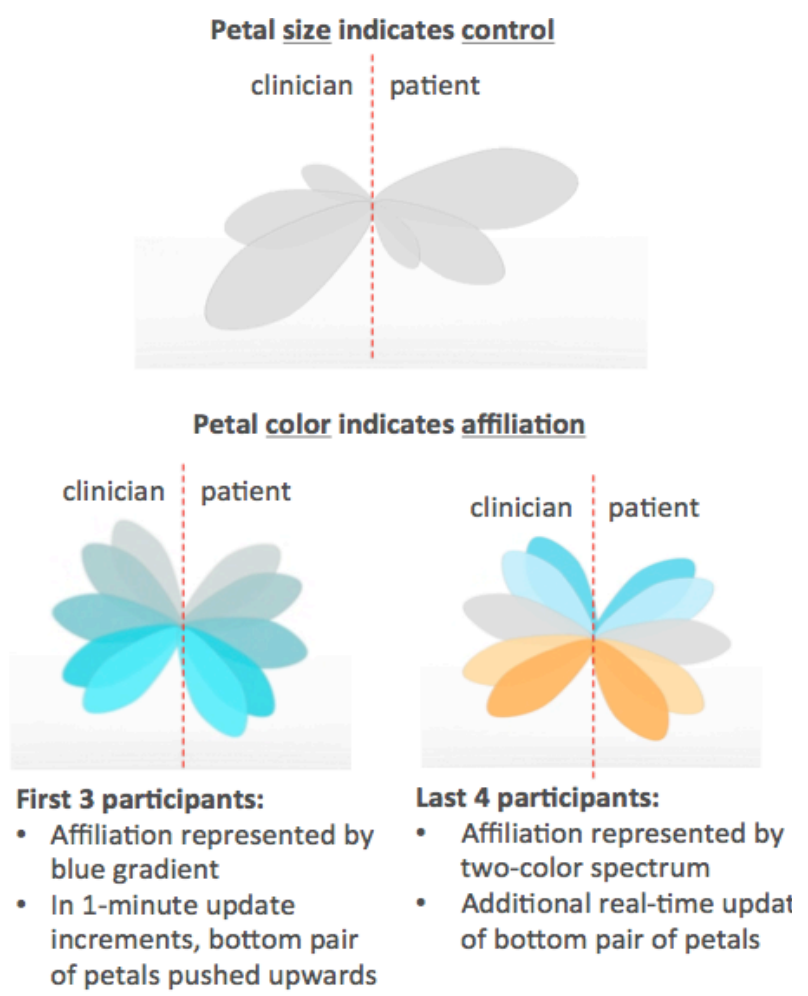

Figure 4. Lotus flower visualization. 
dialogue between two parties while seeing the recent few minutes as well. Each pair of petals represents a segment of time in which nonverbal cues are captured between two people. We designed the lotus to be split in half on a vertical axis, with the clinician's nonverbal signals represented on the left and the patient's on the right (as annotated in Figure 4). After one minute of exchange, a new petal appears on each side at the base of the flower. The size and color of this petal indicates how much or how little control and affiliation each user has displayed in the previous minute.

\section{B. Methods}

(1) Participants: We brought back 7 of the original 16 healthcare professionals who participated in our initial lab study. We purposively selected participants who represented a diversity of opinions and a broad range of specialites. These participants were: 1 physician, 1 dentist, 2 certified nursing assistants, 1 nurse, and 2 emergency medical technicians. Five men and 2 women returned, ranging in age from 25 to 51 years (mean 39.2). All were compensated for their time with a software gratuity. Five returning particpants reported in the initial lab study that they would use the original visualization in a professional setting, one was unsure, and one said she would not. Thus, we had a range of accepting and skeptical participants.

(2) Study Procedures: We conducted follow-up interviews four weeks after the initial lab study. Each participant took part in a 45-minute session. We reconfirmed their responses to the original visualization and introduced the new lotus flower visualization. We trained participants to interpret the new visual feedback by giving them a handout that displayed the meaning of the control and affiliation dimensions and playing a training video showing the full range of petal size and color.

To gather impressions of the visual feedback, we replayed the participants' original role-play video from the previous session on a 24" computer display. Alongside the display of this video, a 30" TV screen simultaneously played back the new visualization seeded with the participant's control and affiliation log data from the lab study. The role-play video included the participant (shot head-on from one camera) and the actor (shot head-on from another camera).

After watching the video and the visual feedback together, we gave participants an acceptability questionnaire that captured the following:

- Likes and dislikes for the composite lotus flower representation

- Reasons for preference for lotus or sun-moon/seesaw

- Whether participants would use the technology in a professional setting (yes/no)

We interviewed participants to draw out implications from their responses and ended the session with a debriefing.

\section{Results}

Six out of 7 participants preferred the lotus flower visualization over the sun-moon and seesaw visualizations. These participants liked seeing the conversation shift over time through changes the lotus flower. The one-minute update rate ended up being too slow to be useful for the first three participants. In addition, those participants had trouble perceiving the differences in color saturation to interpret the affiliation dimension.

These unanimous preferences on update rate and color drove two design changes for the last 4 participants: incorporating a real-time update and changing the saturation gradient from one-color to two. One these changes were made, one of the last 4 participants said that he thought it was "way better" than the sun-moon/seesaw because "it was more fluid, less distracting, subtle, and effective" (P1). He liked seeing the course of a conversation by looking at all the visible petals from the last four minutes, even as the bottom-most petal was changing in real-time. An oncology nurse who viewed the lotus flower visualization with the real-time update thought the petals included "more data to process at a time" (P4) but it would be easy enough for her to understand with more training.

At first, all 7 participants had the same response about use of visual nonverbal communication feedback in a professional setting as they reported in the lab study. But during the interview, P16-who initially did not think she would use Entendre at all-said she could see use by healthcare professionals (particularly in oncology) and mental health counselors who are motivated to be more empathic. She was the only participant who was not enthusiastic about being able to use Entendre as a general training tool in healthcare. She thought that people who were not empathic would need such a tool, and her assumption was that they also would not be motivated to use it. However, the rest of the participants believed that this tool could provide an effective way to learn what nonverbal cues relate to empathy.

In addition, we drew out reactions to our specific design implications: update rate, position of the display, color, and visual metaphor, described in the sections that follow.

(1) Update rate. The update rate proved crucial in determining the acceptability of the lotus visualization. Despite concerns from the lab study participants about potential distractions from the frequent update rate, the first 3 follow-up study participants wanted an instant snapshot of the feedback and did not want to wait a whole minute to see their control and affiliation levels change. P7 felt that the faster update rate from the lab study gave him more information, because "it was hard to remember how the conversation was going in the last minute." An emergency medical technician agreed, saying that with the one-minute update he lost the opportunity to find out "what was I talking about when that [change in feedback] happened” (P3).

Given this unanimous feedback among the first 3 participants, we decided to test a revised version of the lotus 
flower visualization with the last 4 participants. In this new version, the newest petal updated in real time to provide instant feedback. Once this change was made, only P16 had an issue with the update rate. P16 a female certified nursing assistant, had more trouble trusting the actual data in the moment but could still see using the aggregated data visualization in some situations. The remaining 3 participants found the real-time update informative.

(2) Position of the display. We were not able to explore this consideration in depth for this study since we did not deploy WOZ feedback in real time. However, when asked about the ideal size and position of the visual feedback, most participants thought that the large 30" display could make changes easy to detect in a clinical setting, but 2 participants said they thought the 17" display used in the lab study could be just as acceptable. Placement seems to be a matter of room configuration and individual preference.

(3) Color. The first 3 participants found it challenging to differentiate between the five blue saturation levels that we used to represent affiliation (the lowest two levels were not used). Because the difference in hue was so subtle, P8 commented that he did not feel like he was getting enough feedback. To him, the colors represented something that was far more relaxing than the task at hand. Given the first 3 participants' views on color, we changed the color gradient to be more obvious for the remaining 4 participants. We used bright orange (to represent warmth) and bright blue (to represent hostility) at each end of the affiliation spectrum, with a neutral gray in the middle. One satisfied participant commented that the mapping of the new palette to affiliation was quite effective. Nevertheless, P4 said she could easily detect petal size differences to interpret control but still needed to learn what the various colors meant before being able interpret affiliation as quickly.

(4) Visual metaphor. The initial 3 participants all debated about whether the subtle artistry of the lotus flower representation detracted from its purpose as a communication training tool. P8, a male certified nursing assistant, felt that, while the new visualization had a "pleasing appearance and may not interfere with the conversation as much," the lotus flower was not as obvious of a metaphor for communication. He was the only healthcare professional who categorically preferred the sun/moon and seesaw, saying that it was "more objective and obvious to me" (P8). P7, a dentist, suggested that a design with abstract shapes might be easier to focus on for real-time information on control and affiliation levels than the lotus petal representation. Despite this suggestion, he ultimately preferred the lotus visualization over the sun-moon and seesaw, since he preferred interpreting control and affiliation together in a single graphical element.

\section{DISCUSSION}

Results from this work indicate that reflective visual feedback from SSP could encourage empathic nonverbal communication. Entendre could serve as a valuable training tool for patient-clinician interaction. Still, motivation to change - or simply to be aware of - one's empathy level is a key ingredient for training healthcare professionals with Entendre. Over the long term, Entendre could be used to assess communication skills and remind clinicians to maintain and improve empathic nonverbal skills, either intermittently or continuously.

Through our design iterations we learned about the importance of metaphor, color, placement, and real-time update rate for conveying effective reflective feedback. In the future, we could explore other design interventions, such as providing tactile feedback that would be imperceptible to the conversation partner and would avoid the need for a visual display. We are also considering less abstract visualizations that are more familiar, such as line graphs. Learning effects could also impact the use of visual design, which should be examined. For example, the abstract visualization like the lotus flower might take longer to learn, but become less distracting over time with practice. In contrast, simple charts, graphs, or tables might be very easy to learn but be more distracting.

Although our findings are promising, our exploratory study design has some limitations that we will address in future work. First, we employed WOZ as a low-cost technique to drive reflective feedback through a scenario-based lab study. Although this approach enabled us to simulate real-time feedback through a role-play, it provided potential users with feedback that captured only the essence of our perceptions of their nonverbal communication style. Thus, simulated use within a role-play scenario could have only approximated real world use. We are currently building Entendre to analyze audio and visual signals in real-time to drive the visual feedback. Follow-up studies with the complete system in real-world settings will allow users to gauge its utility as well as level of distraction of real-time reflective feedback. In particular, future evaluations will explore the real-world use of Entendre in clinical settings, and could be examined in other settings in which effective interpersonal communication is paramount.

Second, our exploratory work relied on the manual capture of nonverbal cues and adjustment of reflective feedback. While this approach enabled us to easily test Entendre as a proof of concept, manual classification by a researcher could have been biased toward capturing more observable and salient cues that resulted in feedback that reflected only part of the nonverbal communication occurring. In our ongoing work, we are developing a classifier that will automate this process, which we can train and test for accuracy. Through this validating work, we will examine the extent to which Entendre corresponds with other measures of empathic patient-centered communication, such as users' self report and human coding of nonverbal behavior. Because individual variation in communication style and contextual factors (e.g., task versus social orientation of an interaction or depth of relationship), can influence the usefulness and fit of reflective feedback, it is important to account for these issues and examine the ways they shape how Entendre performs.

Despite these limitations, findings from this exploration of Entendre show substantial promise of reflective visual feedback on nonverbal communication for healthcare 
professionals. Not only could Entendre be used as a training tool for real-time feedback, but also captured data could be examined for nonverbal communication skill development over time. Because Entendre uses audio and video streams, this approach could be applied to both face-to-face interactions and remote telehealth interactions. Entendre could also be useful in a range of non-clinical settings in which interpersonal communication is important, such as teaching or videoconferencing.

\section{CONCLUSION}

In our design exploration, we presented a novel approach for reflecting informative feedback on communication style. Using SSP, automated assessment can be particularly valuable for encouraging empathic, patient-centered communication by healthcare professionals. This initial work provides early evidence that reflective visualizations of nonverbal behavior are informative and acceptable to healthcare professionals, given consideration of our design implications. Tools, such as Entendre, could enhance clinicians' empathic patient-centered communication skills and lead to greater patient involvement in health care and improved health outcomes in the future.

\section{Acknowledgments}

We would like to thank the healthcare professionals who participated in our study. We also wish to acknowledge Roland Fernandez and Jessica Herron for production assistance.

\section{References}

[1] S. Lelorain, A. Brédart, S. Dolbeault and S. Sultan, "A systematic review of the associations between empathy measures and patient outcomes in cancer care", Psychooncology, vol. 21, Dec. 2012, p. 1255-64.

[2] S.G. Henry, A. Fuhrel-Forbis, M.A. Rogers and S. Eggly, "Association between nonverbal communication during clinical interactions and outcomes: a systematic review and meta-analysis", Patient Educ Couns, vol. 86, Mar. 2012, p. 297-315.

[3] M.R. DiMatteo, T. Taranta, H.S. Friedman and L.M. Prince, "Predicting patient satisfaction from physicians' nonverbal communication skills", Med Care, vol. 19, 1980, p. 173-387.

[4] K.B. Zolnierek and M.R. DiMatteo, "Physician communication and patient adherence to treatment: a meta-analysis", Med Care, vol. 47, Aug. 2009, p. 826-34.
[5] J. Cegala and S. Lenzmeier Broz, "Physician communication skills training: a review of theoretical backgrounds, objectives and skills", Medical education, vol. 36, 2002, p. 1004-1016.

[6] R.A. Patel, A.L. Hartzler, M. Czerwinski, W. Pratt, A.L. Back, A. Roseway. "Leveraging visual feedback from social signal processing to enhance clinicians' nonverbal skills", Works-in-Progress track in Proceedings of the 2013 ACM conference on Human Factors in Computing, 2013 (in press).

[7] A. Vinciarelli, M. Pantic, H. Bourlard and A. Pentland, "Social signals, their function, and automatic analysis: a survey", Proceedings of the 10th international conference on Multimodal interfaces, 2008, p. 61-68.

[8] A. Pentland, Honest signals: how they shape our world, Cambridge, MA: MIT Press, 2010.

[9] B. Byun, A. Awasthi, P.A. Chou, A. Kapoor, B. Lee and M. Czerwinski, "Honest signals in video conferencing", Multimedia and Expo (ICME), 2011 IEEE International Conference on, 2011, p. 1-6.

[10] T.J. Gallager, P.J. Hartung, S.W. Gregory, "Assessment of a measure of relational communication for doctor-patient interactions.", Patient Educ Couns, vol. 45, Dec. 2001, p.211-8.

[11] J.K. Burgoon, D.B. Buller and W.G. Woodall, Nonverbal communication, Allyn \& Bacon, 2010.

[12] M. Argyle, Bodily communication, New York: Methuen, 1988.

[13] D.J. Kiesler and S.M. Auerbach, "Integrating measurement of control and affiliation in studies of physician-patient interaction: the interpersonal circumplex", Soc Sci Med, vol. 57, Nov. 2003, p. 1707-22.

[14] M.K. Buller and D.B. Buller, "Physicians' communication style and patient satisfaction", J Health Soc Behav, vol. 28, Dec. 1987, p. 375-88.

[15] T. Kim, A. Chang, L. Holland and A.S. Pentland, "Meeting mediator: enhancing group collaborationusing sociometric feedback", Proceedings of the 2008 ACM conference on Computer-supported Cooperative Work, 2008, p. 457-466.

[16] J. Sturm, O.H. Herwijnen, A. Eyck and J. Terken, "Influencing social dynamics in meetings through a peripheral display", Proceedings of the 9th International Conference on Multimodal Interfaces, 2007, p. 263270.

[17] D. Angus, B. Watson, A. Smith, C. Gallois and J. Wiles, "Visualising Conversation Structure across Time: Insights into Effective DoctorPatient Consultations", PLoS One, vol. 7, 2012, p. e38014.

[18] S.W. Mercer, M. Maxwell, D. Heaney and G.C. Watt, "The consultation and relational empathy (CARE) measure: development and preliminary validation and reliability of an empathy-based consultation process measure", Fam Pract, vol. 21, Dec. 2004, p. 699-705.

[19] T.A. D'Agostino and C.L. Bylund, "The Nonverbal Accommodation Analysis System (NAAS): initial application and evaluation", Patient Educ Couns, vol. 85, Oct. 2011, p. 33-9.

[20] D.L. Roter and S. Larson, "The Roter interaction analysis system (RIAS): utility and flexibility for analysis of medical interactions", Patient Educ Couns, vol. 46, 2002, p. 243-251. 